\title{
Bright White-Light-Emitting Device from Ternary Nanocrystal Composites
}

\author{
By Yanqin Li, Aurora Rizzo, Roberto Cingolani, and Giuseppe Gigli*
}

Light-emitting devices (LEDs) based on colloidal inorganic semiconductor nanocrystals (quantum dots, QDs) represent a completely new technology platform for the development of flat-panel displays and flat-panel lighting systems. Their major advantages are the easy tuning of the saturated color emission across the visible-near-IR range and the high chemical and optical stability of the nanocrystal composites. These characteristics open the way to a new class of hybrid devices in which the low-cost, flexible technology of organic LEDs is combined with the long operating lifetime of inorganic semiconductor devices. In the work reported here, we demonstrate the first hybrid white-LED whose emission originates only from the ternary nanocrystal composites, with luminance performances matching the requirements of the lighting industry. Bright white-light emission is obtained from ternary QD composites by controlling the Förster energy transfer and chargetrapping mechanisms between the different components. The proposed approach provides a new general method for the fabrication of stable white-LEDs with a potentially long lifetime.

All-organic white-LEDs have been investigated for their potential applications in the lighting industry and backlighting applications in displays. ${ }^{[1-5]}$ The main advantages of organic technology for white-light generation with respect to other competing technologies, such as GaN-based devices, ${ }^{[6]}$ lie in the possibility of fabricating large-area, perhaps flexible, lightemitting panels by low-cost fabrication techniques, such as evaporation and spin-casting. ${ }^{[7-11]}$ Inorganic LEDs are, in fact, intrinsically point sources requiring complicated and expensive technological processes when integration in diffuse sources is required. To obtain white light from organic systems, different approaches are commonly used, namely, evaporation or co-evaporation of multilayer structures, ${ }^{[7-9]}$ spincasting of different light-emitting compounds in a single active-layer structure ${ }^{[10,11]}$ exploitation of exciplex emission, ${ }^{[12]}$ and, recently, the synthesis of single white-light-emitting molecules. ${ }^{[13]}$ However, in most of these approaches, the purity of the color emission is strongly affected by the different aging rates of the active compounds. ${ }^{[7,8]}$ In addition, device lifetimes are short, owing to heating, at the high luminance values re-

[*] Prof. G. Gigli, Dr. Y. Q. Li, A. Rizzo, Prof. R. Cingolani National Nanotechnology Laboratory (NNL) of CNR-INFM Distretto Tecnologico ISUFI

Engineering of Innovation Department, Lecce University Via Arnesano Km 5, 73100 Lecce (Italy)

E-mail: giuseppe.gigli@unile.it quired for lighting applications $\left(>1000 \mathrm{~cd} \mathrm{~m}^{-2}\right)$. In this scenario the integration of QDs into organic LEDs has the potential to overcome most of these problems, and it turns out to be a rich field of scientific endeavor. ${ }^{[14-19]}$ The broadly tunable, saturated-color emission performance of QDs is unsurpassed by any class of organic chromophores. Furthermore, the environmental stability of covalently bonded inorganic nanocrystals is expected to increase the device lifetimes at the high luminance/current regimes required for lighting. Moreover, the high quantum yield and narrow band emission of QDs satisfy the technological requirements of flat panel displays and imaging applications. Coe et al. ${ }^{[17,18]}$ have demonstrated a hybrid high-efficiency $\left(1.6 \mathrm{~cd} \mathrm{~A}^{-1}\right.$ at $\left.2000 \mathrm{~cd} \mathrm{~m}^{-2}\right)$ monochromic LED by exploiting phase segregation processes between CdSe quantum dots and an organic matrix. This resulted in the formation of a very thin layer of CdSe QDs sandwiched between the organic layers, which improved the electron injection in the inorganic active medium and, in turn, the LED performances. However, so far, few studies have been reported on white-LEDs based on QDs. Here, we demonstrate the first efficient hybrid LED with near-white light emission from chemically and optically stable ternary nanocrystal composites dispersed in an organic matrix. Bright white-light emission is obtained from homogeneous blends, without phase segregation between the active ternary QD composites and the organic matrix, exploiting the energy-transfer and chargetrapping properties of the different species. The proposed approach is a new general method for the fabrication of highefficiency white-LEDs with potentially long operating lifetimes.

The key element in a high-performance hybrid device in which QDs act as lumophores is the occurrence of efficient exciton recombination in the inorganic nanocrystals. This is usually inhibited by the poor electron conduction of the inorganic species, which limits exciton formation. ${ }^{[20,21]}$ Therefore low electroluminescence (EL) efficiency is observed in QD/ polymer blend-based devices. ${ }^{[16,22,23]}$ The phase-segregation technique proposed by Coe et al. allows the fabrication of high-efficiency hybrid monochromatic emission devices, but it involves a narrow QD size distribution to form high-coverage monolayers, which are required for monochromatic-emitting QD-LEDs. ${ }^{[17,24]}$ To date, efficient QD-LEDs that emit white light from the nanocrystals alone are still a challenge owing to the lack of proper fabrication techniques. To obtain white light, all the primary colors (blue B, green G, and red R) have to be produced simultaneously. In order to obtain efficient simultaneous emission from different sizes of QD composites, 
we propose a novel device structure in which exciton formation in the inorganic QDs is not exclusively obtained by direct charge injection but by the precise control of the energy/ charge transfer mechanisms from the organic host. The structure of the device and the emission mechanisms are shown in Figures 1a and c. As a control, another device structure (Fig. 1b) with binary QD composites was made.

The absorption and photoluminescence (PL) spectra of $4,4^{\prime}, N, N^{\prime}$-diphenylcarbazole (CBP) and QDs (B, G, R) in chloroform solution are shown in Figure $2 \mathrm{a}$. The luminescence of CBP shows significant spectral overlap with the absorption spectra of all our QDs, allowing the possibility of efficient exciton energy transfer from CBP to ternary QD composites, according to Förster theory. ${ }^{[25]}$ Evidence of Förster energy transfer from polymers to colloidal $\mathrm{CdSe} / \mathrm{ZnS}$ core-shell quantum dots has been recently reported by Anni et al. ${ }^{[26]}$ A clear spectral overlap between the emission and absorption of different-size QDs is also observed, showing that Förster energy transfer between different nanocrystals is a further possible pathway. This is in agreement with the work of Bawendi and co-workers, ${ }^{[27]}$ in which spectroscopic evi-

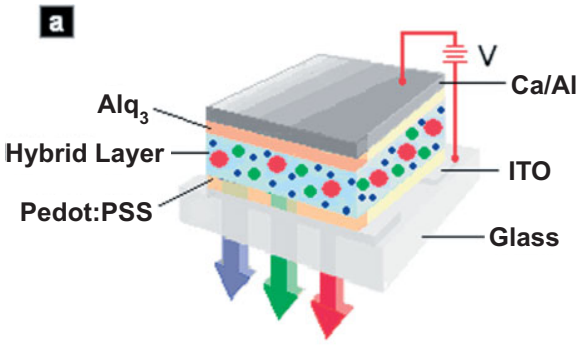

Light Output

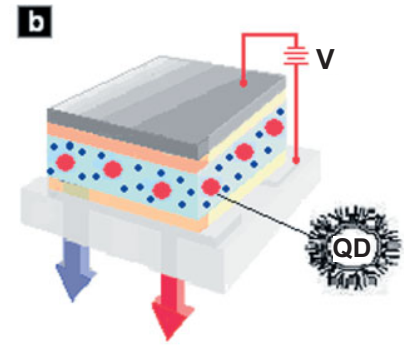

Light Output

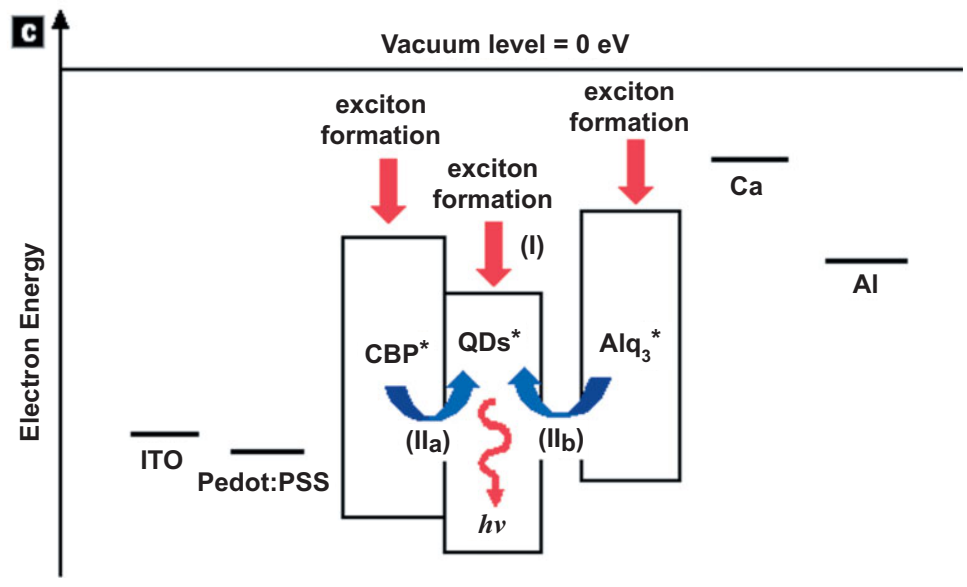

Figure 1. The structures and simplified energy level diagram of devices and possible exciton creation mechanisms. a) The structure of the device ITO//PEDOT:PSS// CBP:QDs (B, G, R)//Alq $q_{3} / / \mathrm{Ca} / \mathrm{Al}$. b) The structure of the device ITO//PEDOT:PSS// CBP:QDs (B, R)//Alq $3 / / \mathrm{Ca} / \mathrm{Al}$. c) Proposed simplified energy level diagram of devices and possible exciton creation mechanisms on the QDs: I) charge trapping and II) Förster energy transfer. ITO: indium tin oxide; PEDOT:PSS: poly(3,4-ethylenedioxythiophene):poly(styrene sulfonate); $4,4^{\prime}, N, N^{\prime}$-diphenylcarbazole; Alq $_{3}$ : tris (8-hydroxyquinoline) aluminium. dence of energy transfer in close-packed CdSe QD solids due to dipole-dipole interdot interactions between proximal dots has been demonstrated. In order to obtain white EL, the QDs' relative molar concentrations were varied, taking into account the emission efficiency of each species. The optimized blend with relative molar concentration CBP/QDs(B)/ QDs (G)/QDs (R) of 4000:18:2:1 [mol \%] resulted in white emission with Commission Internationale d'Eclairage (CIE) coordinates $(0.32,0.45)$. PL and photoluminescence excitation (PLE) measurements were performed on films of such a blend (Fig. 2b).

For an excitation wavelength of $340 \mathrm{~nm}$, four emission bands, at 377, 490, 540, and $615 \mathrm{~nm}$, originating from the CBP and QDs $(B, G, R)$, respectively, are observed. The weakness of the QD bands evidences the excessive CBP concentration, whereas the ratio between the luminescence intensity of the different QDs evidences energy transfer from the small to the large dots and an excessive blue-dot concentration. PLE spectra with detection wavelengths set at 377, 490, 540, and $615 \mathrm{~nm}$, corresponding to the emission peaks of CBP and QDs $(B, G, R)$, respectively, overlap the absorption of CBP, showing that the emission of QDs originates mainly from the excitation energy transferred from CBP.

Electroluminescence measurements have been carried out on the following LED structure: ITO//PEDOT:PSS//CBP:QDs (B, G, R)// $\mathrm{Alq}_{3} / / \mathrm{Ca} /$ $\mathrm{Al}$ (see Fig. 1a), where ITO is indium tin oxide; PEDOT:PSS, poly(3,4-ethylenedioxythiophene):poly(styrene sulfonate); and $\mathrm{Alq}_{3}$, tris(8-hydroxyquinoline) aluminum. The EL spectrum at $13 \mathrm{~V}$ is shown in Figure 3a. Spectral peaks at wavelengths of 490, 540, and $618 \mathrm{~nm}$ are attributed to the blue-, green-, and red-light-emitting quantum dots, respectively, in agreement with the PL spectra of the isolated QDs in the solid state (see Fig. 3b). Unlike the PL spectrum (see Fig. 2b), a complete quenching of the CBP band is observed in the EL spectrum, resulting in efficient emission of the three primary colors from the QDs, giving rise to a balanced near-white overall emission (CIE $(0.32,0.45))$ at $13 \mathrm{~V}$. Furthermore, the color coordinates do not change markedly when the operating voltage is changed in the range 10-28 V. This suggests that in the EL spectrum a novel and more efficient transfer mechanism from the host material $\mathrm{CBP}$ and the electron transfer layer $\mathrm{Alq}_{3}$ to the lower-energy light-emitting species QDs occurs. Possible pathways leading to emissive states are shown in Figure 1c. In our device (see the proposed simplified energy diagram ${ }^{[23,28]}$ in Fig. 1c), holes are injected from the ITO contact through the PEDOT:PSS layer into the CBP host matrix, and are transported towards the QDs. Similarly, electrons are injected from the $\mathrm{Ca} / \mathrm{Al}$ cathode into the $\mathrm{Alq}_{3}$ and are transported to the QDs. Then ex- 

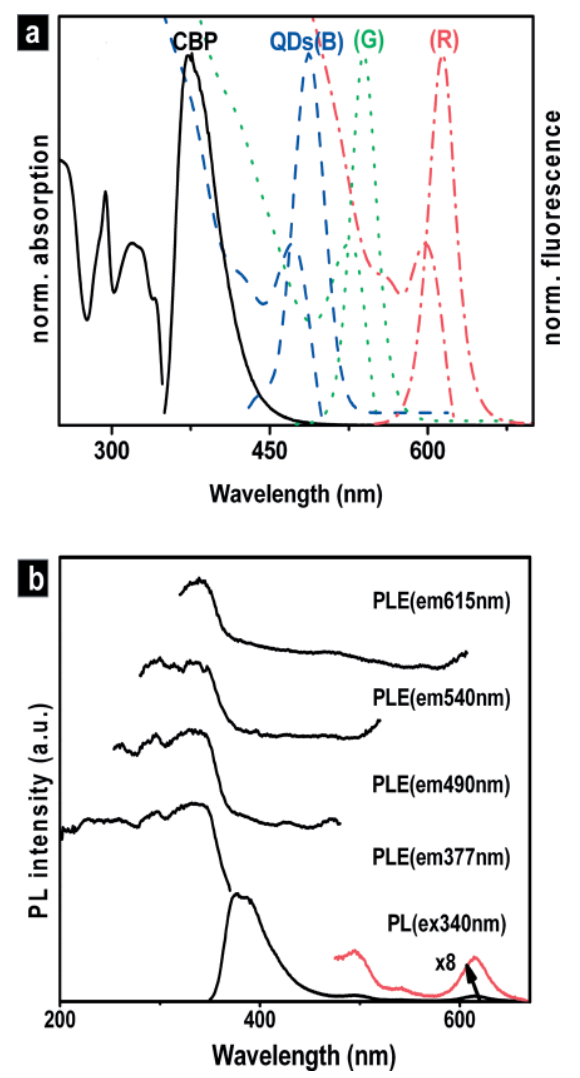

Figure 2. Optical properties of the samples in solution and as film. a) Absorption (left) and photoluminescence (PL, right) spectra for CBP (solid line), QDs (B) (dashed), QDs (G) (dotted), and QDs (R) (dashed dotted) in chloroform. b) PL and PL efficiency (PLE) spectra of the blend film CBP/QDs $(B, G, R)$ with the ratio CBP/QDs (B)/QDs (G)/ QDs $(R)=4000: 18: 2: 1[\mathrm{~mol} \%]$.

citon generation on QDs occurs via two parallel processes: I) charge trapping and II) Förster energy transfer from CBP and $\mathrm{Alq}_{3}$. In the former process, electrons may be trapped in the QDs, owing to the relative energy alignment of the lowest un- occupied molecular orbital (LUMO) levels of CBP, $\mathrm{Alq}_{3}$, and QDs. Thus, efficient exciton formation on the QDs can occur after recombination with high-mobility holes. In the latter process, excitons form on organic molecules CBP $\left(\mathrm{II}_{\mathrm{a}}\right)$ and $\mathrm{Alq}_{3}\left(\mathrm{II}_{\mathrm{b}}\right)$, then undergo Förster energy transfer to the lowerenergy QD sites, where they can recombine radiatively. The different EL and PL spectra evidence that in our hybrid QD-LEDs charge trapping is a dominant process for exciton formation, in agreement with the poor charge conduction properties of $\mathrm{CdSe} / \mathrm{ZnS}$ core/shell QDs, ${ }^{[20,21]}$ and the excellent electron injection/transfer properties of $\mathrm{Alq}_{3}$. In order to achieve white EL the different color light emissions from QDs have to be accurately balanced by controlling both the Förster energy transfer and charge-trapping mechanisms, in relation to QD PL efficiency and concentration. In Figure $3 \mathrm{c}$ voltage-current $(V-I)$ and voltage-luminance $(V-L)$ characteristics for the device ITO//PEDOT:PSS// CBP:QDs $(\mathrm{B}, \mathrm{G}, \mathrm{R}) / / \mathrm{Alq}_{3} / / \mathrm{Ca} / \mathrm{Al}$ are reported. The maximum brightness of the device is $1050 \mathrm{~cd} \mathrm{~m}^{-2}$ at $58 \mathrm{~mA} \mathrm{~cm}^{-2}$, which corresponds to a current efficiency of $1.8 \mathrm{~cd} \mathrm{~A}^{-1}$; a turn-on voltage of $6 \mathrm{~V}$ is measured in air. To our knowledge, this is the highest efficiency hybrid device with white-light emission from ternary QD composites alone whose luminance satisfies lighting application requirements (i.e., $1000 \mathrm{~cd} \mathrm{~m}^{-2}$ ). As a control, a device structure (Fig. 1b) with only blue- and red-lightemitting QDs has been fabricated. The EL spectrum reported in Figure 4a demonstrates that the emission is only from QD composites; $\mathrm{Alq}_{3}$ functions only as an electron-injection and transfer layer. The $V-I-L$ characteristics for the binary device are shown in Figure $4 \mathrm{~b}$. The maximum brightness of the device is $1500 \mathrm{~cd} \mathrm{~m}^{-2}$ at $62 \mathrm{~mA} \mathrm{~cm}^{-2}$, which corresponds to a current efficiency of $2.2 \mathrm{~cd} \mathrm{~A}^{-1}$.

In conclusion, hybrid white-LEDs have been fabricated using three $\mathrm{CdSe} / \mathrm{ZnS}$ QDs of different size blended in a CBP organic matrix. Accurate control and balance of the Förster energy and charge-trapping processes between the different active components has allowed the first high-efficiency hybrid device with emission originating only from the inorganic com-
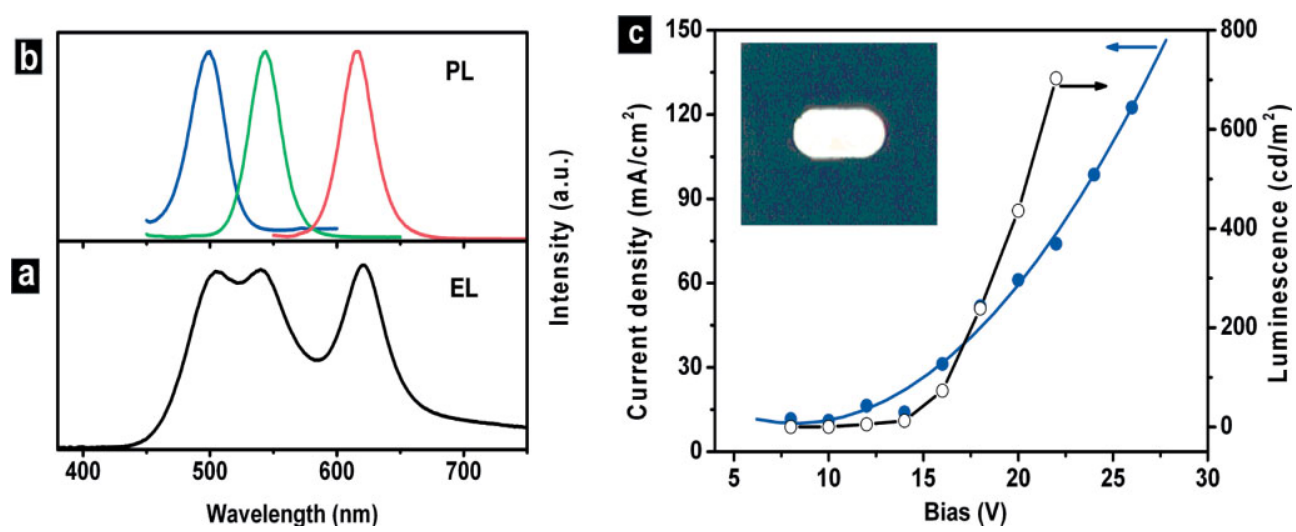

Figure 3. EL and PL spectra and characteristics of the ternary QD device. a) EL spectrum for the device ITO//PEDOT:PSS//CBP:QDs (B, G, R, mol \% = 18:2:1)//Alq $q_{3} / / \mathrm{Ca} / \mathrm{Al}$. b) Photoluminescence spectra corresponding to isolated blue, green, and red QDs, measured in the solid state. C) Current density-voltage $(\mathbf{O})$ and current-luminescence $(\bigcirc)$ characteristics of the device. Inset: A photograph of the working device. 

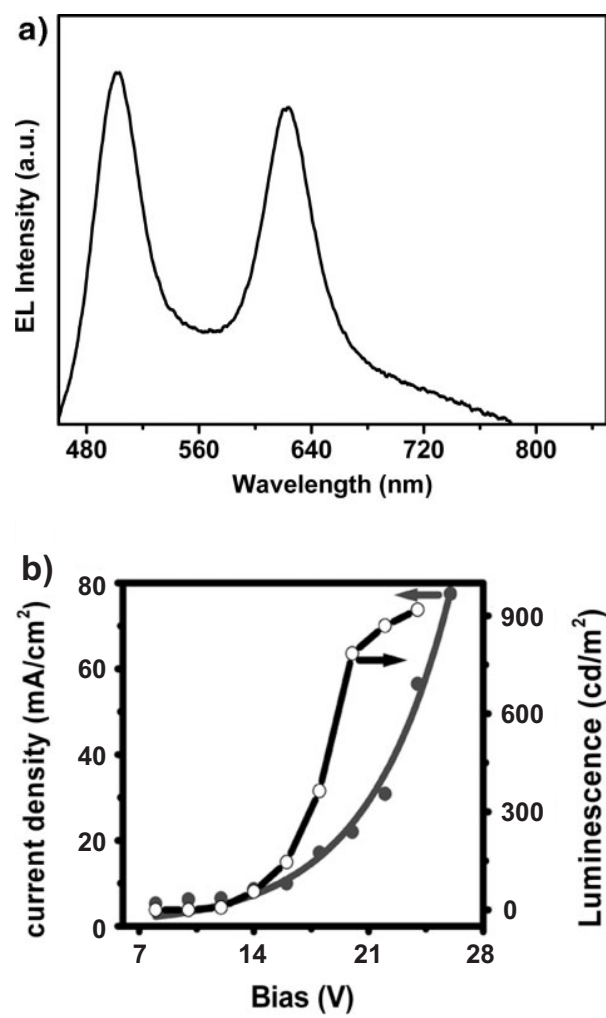

Figure 4. a) EL spectrum and characteristics of the binary QD device. Main panel: EL spectrum of the device ITO//PEDOT:PSS// CBP:QDs (B, R, c\% = 18:1)//Alq $q_{3} / / \mathrm{Ca} / \mathrm{Al}$. b) $V-I(O)$ and $V-L(O)$ characteristics of the device.

ponents to be fabricated. The proposed device provides a potential and general method of fabricating long-lifetime EL devices combining the advantages of organic technology in terms of cost and flexibility with those of inorganic materials in terms of stability. Color coordinates and light quality can be optimized by further fine control of the QD composites.

\section{Experimental}

Core/shell CdSe/ZnS QDs were from Evident Technologies, Inc. The nanocrystal quantum dots were enhanced with a proprietary coating technology and TOPO (trioctylphosphine oxide) surfactant, which improves their stability and brightness. We selected three of them for hybrid white-LED studies (Lake Placid blue QDs with crystal diameter $1.89 \mathrm{~nm}$ (B), Catskill green QDs with crystal diameter $2.37 \mathrm{~nm}$ $(\mathrm{G})$, and maple-red orange QDs with crystal diameter $5.11 \mathrm{~nm}(\mathrm{R})$ ).

Fabrication of Hybrid EL Devices: Devices consisting of ITO// PEDOT:PSS//CBP:CdSe/ZnS (B, G, R)//Alq $3 / / \mathrm{Ca} / \mathrm{Al}$ were fabricated as follows: A hole-transporting layer $(100 \mathrm{~nm})$ of PEDOT:PSS, used to lower the hole injection barrier at the ITO surface, was spin-deposited onto the cleaned ITO-coated glass substrate $(120 \mathrm{~nm}, 15 \Omega / \square)$. The layer was then heated at $110^{\circ} \mathrm{C}$ for $10 \mathrm{~min}$ to remove the residual water solvent. Then a layer of $\mathrm{CdSe} / \mathrm{ZnS}$ QDs $(\mathrm{B}, \mathrm{G}, \mathrm{R})$ and $\mathrm{CBP}$ blend $(80 \mathrm{~nm})$ was spin-coated from a chloroform solution on the surface of the PEDOT:PSS. Finally, a $15 \mathrm{~nm}$ thick layer of $\mathrm{Alq}_{3}$, acting as an electron-injecting/transferring and exciton-donor material, and a $50 \mathrm{~nm}$ thick Ca cathode covered with a $150 \mathrm{~nm}$ thick Al layer were deposited by thermal evaporation at a pressure of $4.0 \times 10^{-6} \mathrm{mbar}$
(1 mbar=100 Pa). A reference device with ITO//PEDOT:PSS// CBP:CdSe/ZnS (B, R)//Alq $3 / / \mathrm{Ca} / \mathrm{Al}$ was fabricated.

Characterization: PL and PLE measurements were performed on thin films and $\mathrm{CHCl}_{3}$ solutions using a Cary Eclipse fluorescence spectrophotometer with an intense xenon flash lamp. Absorption measurements were carried out using a Cary 5000 UV-vis spectrophotometer. EL spectra were measured by a spectroradiometer OL 770 (Optronic Laboratories). Voltage-luminance-current characteristics were measured using a Keithley source measurement unit (Keithley 2700 and 2400) with an amplified silicon photodiode (RS 303-674, Hamamatsu). All the measurements were performed at room temperature in air without encapsulation. The device worked well without degradation or dark points during the working time.

Received: January 27, 2006

Final version: April 6, 2006

Published online: September 14, 2006

[1] B. W. D'Andrade, M. E. Thompson, S. R. Forrest, Adv. Mater. 2002 $14,147$.

[2] X. H. Zhang, M. W. Liu, O. Y. Wong, C. S. Lee, H. L. Kwong, S. T. Lee, S. K. Wu, Chem. Phys. Lett. 2003, 369, 478.

[3] K. O. Cheon, J. Shinar, Appl. Phys. Lett. 2002, 81, 1738.

[4] J. Thompson, R. I. R. Blyth, M. Mazzeo, M. Anni, G. Gigli, R. Cingolani, Appl. Phys. Lett. 2001, 79, 560.

[5] G. Cheng, Y. Zhao, Y. F. Zhang, S. Y. Liu, F. He, H. Q. Zhang, Y. G. Ma, Appl. Phys. Lett. 2004, 84, 4457.

[6] J. W. Orton, C. T. Foxon, Rep. Prog. Phys. 1998, 61, 1.

[7] G. Li, J. Shinar, Appl. Phys. Lett. 2003, 83, 5359.

[8] R. S. Deshpande, V. Bulovic, S. R. Forrest, Appl. Phys. Lett. 1999, $75,888$.

[9] C. H. Kim, J. Shinar, Appl. Phys. Lett. 2002, 80, 2201.

[10] M. Granström, O. Inganäs, Appl. Phys. Lett. 1996, 68, 147.

[11] M. Anni, G. Gigli, V. Paladini, R. Cingolani, G. Barbarella, L. Favaretto, G. Sotgiu, M. Zambianchi, Appl. Phys. Lett. 2000, 77, 2458.

[12] M. Mazzeo, D. Pisignano, F. Della Sala, J. Thompson, R. I. R. Blyth, G. Gigli, R. Cingolani, G. Sotgiu, G. Barbarella, Appl. Phys. Lett. 2003, 82, 334.

[13] M. Mazzeo, V. Vitale, F. Della Sala, M. Anni, G. Barbarella, L. Favaretto, G. Sotgiu, R. Cingolani, G. Gigli, Adv. Mater. 2005, 17, 34.

[14] J. L. Zhao, J. Y. Zhang, C. Y. Jiang, J. Bohnenberger, T. Basché, A. Mews, J. Appl. Phys. 2004, 96, 3206.

[15] H. Yang, P. H. Holloway, J. Phys. Chem. B 2003, 107, 9705.

[16] M. C. Schlamp, X. G. Peng, A. P. Alivisatos, J. Appl. Phys. 1997, 82, 5837.

[17] S. Coe, W. K. Woo, M. Bawendi, V. Bulovic, Nature 2002, 420, 800.

[18] S. Coe, W. K. Woo, J. S. Steckel, M. Bawendi, V. Bulovic, Org. Electron. $\mathbf{2 0 0 3}, 4,123$.

[19] Y. Q. Li, A. Rizzo, M. Mazzeo, L. Carbone, L. Manna, R. Cingolani, G. Gigli, J. Appl. Phys. 2005, 97, 113501.

[20] C. A. Leatherdale, C. R. Kagan, N. Y. Morgan, S. A. Empedocles, M. A. Kastner, M. G. Bawendi, Phys. Rev. B 2000, 62, 2669.

[21] N. C. Greenham, X. G. Peng, A. P. Alivisatos, Phys. Rev. B 1996, 54 , 17628.

[22] B. O. Dabbousi, M. G. Bawendi, O. Onitsuka, M. F. Rubner, Appl. Phys. Lett. 1995, 66, 1316.

[23] H. Mattoussi, L. H. Radzilowski, B. O. Dabbousi, E. L. Thomas, M. G. Bawendi, M. F. Rubner, J. Appl. Phys. 1998, 83, 7965.

[24] S. Coe, J. S. Steckel, W. K. Woo, M. G. Bawendi, V. Bulovic, $A d v$. Funct. Mater. 2005, 15, 1117 .

[25] J. R. Lakowicz, Principles of Fluorescence Spectroscopy, Plenum, New York 1983.

[26] M. Anni, L. Manna, R. Cingolani, D. Valerini, A. Cretí, M. Lomascolo, Appl. Phys. Lett. 2004, 85, 4169.

[27] C. R. Kagan, C. B. Murray, M. G. Bawendi, Phys. Rev. B 1996, 54, 8633.

[28] I. G. Hill, A. Kahn, J. Appl. Phys. 1998, 84, 5583 